\title{
Procedure End Relative to Reference Time Point
}

National Cancer Institute

\section{Source}

National Cancer Institute. Procedure End Relative to Reference Time Point. NCI

Thesaurus. Code C117541.

An indication or description that the end of the procedure is before, during or after a reference moment in time. 